\title{
Tendência temporal de tabagismo em população urbana: um estudo de base populacional no Sul do Brasil
}

\author{
Time trends in smoking in an urban population: \\ a population-based study in Southern Brazil
}

\author{
1 Programa de Pós-graduação \\ em Epidemiologia, \\ Universidade Federal de \\ Pelotas, Pelotas, Brasil. \\ 2 Divisão de População \& \\ Saúde, Universidade Federal \\ do Rio Grande, Rio Grande, \\ Brasil. \\ Correspondência \\ J. L. Dias-Damé \\ Programa de Pós-graduação \\ em Epidemiologia, \\ Universidade Federal de \\ Pelotas. \\ Rua Marechal Deodoro 1160, \\ 3 o piso, Pelotas, RS \\ 96020-220, Brasil. \\ josianeddame@yahoo.com.br
}

\begin{abstract}
This study evaluated time trends in smoking prevalence according to gender and family income among individuals 20 years or older in Pelotas, Rio Grande do Sul State, Brazil. Five population-based surveys using census tracts were conducted in the city from 2002 to 2010. Smoking was defined as consumption of one or more cigarettes per day for at least one month. Time trend was assessed using the chi-square test for linear trend. 15,136 individuals were enrolled in these surveys. During this period, overall smoking prevalence decreased from $28 \%$ (25.8-30.4) in 2002 to $21 \%$ (19.5-23.5) in 2010. This 23\% decline was similar in both genders, but differed significantly according to family income (smoking prevalence increased as income dropped). From 2002 to 2010, smoking decreased by $26 \%$ in the lowest income quintile and $39 \%$ in the highest. Despite such reductions, smoking prevalence is still high, indicating the need to boost control measures, especially among low-income groups.
\end{abstract}

Smoking; Health Surveys; Urban Population; Prevalence
Josiane L. Dias-Damé 1

Juraci A. Cesar 1,2

Suele Manjourany Silva 1

\section{Introdução}

O tabagismo é a principal causa de morte para metade dos seus usuários, com $50 \%$ deles indo a óbito dos 35 aos 69 anos de idade, ou seja, no seu período de maior produtividade. Nenhum outro fator de risco é tão devastador quanto o cigarro. O número de óbitos decorrentes do tabagismo supera com larga margem aqueles atribuídos à AIDS, uso de drogas (lícitas e/ou ilícitas), acidentes de trânsito, assassinatos e suicídios somados 1. Se forem mantidas as tendências atuais, em 2030 o tabaco será responsável por cerca de 8 milhões de óbitos por ano em todo o mundo, com $80 \%$ destas mortes ocorrendo de forma prematura entre aqueles residentes em países de baixa e média renda 2 .

Os custos decorrentes do uso do tabaco são enormes para o indivíduo, para a família e para a sociedade. Esses custos incluem o valor gasto na compra dos cigarros, a perda de renda em virtude das despesas com doenças, morte prematura e doenças impostas àqueles afetados pelo tabagismo passivo 1,3. Estudo realizado em 15 capitais brasileiras e no Distrito Federal revelou que $13,6 \%$ das mortes ocorridas em 2003 de pessoas com 35 anos ou mais tinham como causa básica o tabagismo, representando 419.435 anos potenciais de vida perdidos no período 4 .

Há, contudo, tendência de diminuição do tabagismo entre os homens em todo o mundo, 
o que tem sido mais evidente entre aqueles de melhor nível socioeconômico. Entre as mulheres, isso também tem ocorrido, mas somente nos países industrializados 5 .

Estudos transversais de base populacional que avaliem a tendência de tabagismo são escassos tanto no Brasil, como em outros países. As diferenças metodológicas entre os estudos de prevalência dificultam a comparação dos dados obtidos nas diferentes pesquisas. Não há consenso na definição de tabagismo, além de haver diferenças quanto à faixa etária, tipo de delineamento, processo amostral, representatividade do estudo, periodicidade da coleta de dados, características da população estudada, taxa de respondentes, dentre outros critérios 6,7.

De 2001 a 2010, foram realizados em Pelotas, Rio Grande do Sul, cinco inquéritos utilizando a mesma definição de tabagismo, mesma faixa etária e delineamento transversal, com intervalos de, no máximo, dois anos, alta taxa de respondentes $(89,3 \%$ a $96,5 \%)$, amostra representativa selecionada a partir de conglomerados e estratificada por nível socioeconômico 7 .

Este estudo teve por objetivo comparar as prevalências de tabagismo entre 2001 e 2010, conforme quintil de renda familiar, faixa etária e sexo, para indivíduos com 20 anos ou mais residentes na zona urbana de Pelotas.

\section{Métodos}

Inquéritos populacionais abrangendo aspectos relacionados à saúde têm sido conduzidos bienalmente em Pelotas, desde 1999. Este município, localizado na metade sul do Estado do Rio Grande do Sul, conta com cerca de 350 mil habitantes, $96 \%$ deles residindo na cidade.

Os inquéritos foram conduzidos na área urbana do município pelo Programa de Pós-graduação em Epidemiologia da Universidade Federal de Pelotas (PPGE/UFPel), mediante consórcio realizado por mestrandos nos biênios 1999/2000, 2001/2002, 2003/2004, 2005/2006, 2007/2008 e 2009/2010. Os dados utilizados nesta análise não incluem aqueles referentes ao consórcio 1999/2000 em virtude de este ter utilizado definição de tabagismo diferente dos demais inquéritos. Maiores detalhes sobre a metodologia empregada nesses inquéritos podem ser obtidos em outra publicação ${ }^{8}$.

A coleta de dados dos inquéritos em questão ocorreu em diferentes períodos: no consórcio de 2001/2002, de fevereiro a maio de 2002; nos de 2003/2004 e 2005/2006, nos meses de outubro a dezembro de 2003 e 2005, respectivamente; no consórcio de 2007/2008, de outubro de 2007 a janeiro de 2008; no de 2009/2010, de janeiro a junho de 2010.

A população-alvo desses inquéritos foi constituída por indivíduos com 20 anos ou mais residentes na área urbana do município por ocasião da coleta de dados. Foram excluídos indivíduos fisicamente e/ou mentalmente incapacitados de responder ao questionário e aqueles institucionalizados (asilos e presídios).

Considerando-se as prevalências de tabagismo observadas nos estudos, que variaram de $27,9 \%$ em 2002 a 21,3\% em 2010, margem de erro aceitável de 2,5 pontos percentuais, $10 \%$ para perdas e recusas e efeito de delineamento de 2,0, o maior "n" necessário seria de 2.712 indivíduos por inquérito. No total de inquéritos conduzidos ao longo do período, esse tamanho de amostra foi passível de obtenção.

O processo amostral foi o mesmo em todos os inquéritos. Foi realizado em dois estágios, com base em conglomerados, tendo como unidades amostrais primárias os setores censitários (áreas delimitadas, compostas por aproximadamente 300 domicílios cada) definidos pelo Instituto Brasileiro de Geografia e Estatística (IBGE) e como unidades amostrais secundárias os domicílios. Os setores foram sorteados sistematicamente, com probabilidade proporcional ao número de domicílios; estes foram selecionados a partir de estratificação por renda familiar média dos chefes de família, utilizando-se dados do IBGE. Em cada setor sorteado, os domicílios foram amostrados de forma sistemática, e, por sua vez, naqueles incluídos no estudo, todos os indivíduos com 20 anos ou mais eram elegíveis.

O tabagismo foi coletado de maneira padronizada em todos os inquéritos, sendo considerados tabagistas tanto os indivíduos que fumavam um ou mais cigarros por dia há pelo menos um mês, quanto os que haviam deixado de fumar há menos de um mês. Foram considerados ex-tabagistas indivíduos que haviam deixado de fumar há mais de um mês 9 .

As variáveis de exposição estudadas foram: sexo (masculino ou feminino), idade (coletada na forma discreta e posteriormente categorizada em 20-29, 30-39, 40-49, 50-59 e 60 ou mais), cor da pele (observada pelo entrevistador e categorizada como branca e parda/preta), escolaridade (coletada na forma discreta em anos completos e categorizada em 0-4, 5-8, 9-11 e 12 ou mais) e renda familiar [coletada na forma contínua em reais $(R \$)$ e, posteriormente, categorizada em quintis].

Para a coleta de dados foram recrutadas candidatas com 18 anos ou mais, que receberam treinamento teórico e prático. Ao final do treinamento, foram selecionadas entre 28 e 38 entrevis- 
tadoras, dependendo do inquérito. Estas foram responsáveis pela coleta dos dados por meio de entrevistas domiciliares. Os indivíduos elegíveis não encontrados após, pelo menos, três visitas em diferentes horários foram considerados como perdas.

O controle de qualidade foi realizado por supervisores do trabalho de campo, em revisita e aplicação de um questionário reduzido para $10 \%$ dos entrevistados. Essa etapa ocorreu com um intervalo de aproximadamente duas semanas após a entrevista inicial.

Os estudos foram submetidos e aprovados pelo Comitê de Ética em Pesquisa da Faculdade de Medicina da UFPel. Todos os participantes assinaram um Termo de Consentimento Livre e Esclarecido antes de dar início às entrevistas. Foram resguardados o direito de não participação na pesquisa e o sigilo acerca das informações obtidas.

As análises foram realizadas para a população geral e estratificadas por sexo. Utilizou-se a estatística descritiva para o cálculo de proporções e respectivos intervalos de 95\% de confiança (IC95\%) para as variáveis estudadas. A fim de se avaliar a tendência entre os quintis de renda em cada inquérito e a tendência para renda e idade no período estudado, foi utilizado o teste qui-quadrado de tendência linear para proporções. Para a avaliação de diferenças entre grupos de idade e renda dentro de cada inquérito, assim como entre os percentuais de redução no período, utilizou-se o teste qui-quadrado de heterogeneidade.

\section{Resultados}

Na Tabela 1, verifica-se que o tamanho da amostra variou de 2.732 a 3.182 participantes, e que as perdas estiveram entre $3,5 \%$ e $10,7 \%$. Dentre as perdas, $56 \%$ ocorreram entre homens e $44 \%$, entre as mulheres.

No total, os inquéritos incluíram 15.136 indivíduos com 20 anos ou mais. Eram, em sua maioria, do sexo feminino (57\%), de cor da pele branca $(82 \%)$, com, pelo menos, oito anos de escolaridade (53\%) e com mais de 40 anos de idade (55\%).

Na Tabela 2, observa-se que a prevalência geral de tabagismo passou de 28\% em 2002 para $21 \%$ em 2010, o que indica redução de $23 \%$. Em relação à renda familiar, verificou-se, em todos os inquéritos, que a prevalência de tabagismo foi, em geral, maior nas categorias de menor quintil de renda (os mais pobres). Ao se avaliarem as prevalências para cada quintil de renda entre 2002 e 2010, verificou-se que essa redução não foi uniforme. Por exemplo, a redução no menor quintil de renda ao longo do período foi de $26 \%$, enquanto no quintil de maior renda (os mais ricos) foi de $39 \%(\mathrm{p}<0,001)$. Em relação à idade, observou-se, neste intervalo, tendência de diminuição da prevalência de tabagismo entre indivíduos com idade inferior a 50 anos. A maior redução ocorreu na faixa etária de 30-39 anos (29\%). Observando-se cada um dos inquéritos, constatou-se que as maiores prevalências de tabagismo ocorreram no grupo de indivíduos com idade entre 40 e 49 anos. Em resumo, até esta faixa etária a prevalência de tabagismo aumentou em todos os inquéritos, ao passo que, a partir dela, houve diminuição.

As Tabelas 3 e 4 mostram a tendência de tabagismo estratificada por sexo. As prevalências de tabagismo nos sexos masculino e feminino passaram de $33 \%(30,0-36,4)$ e $24 \%(21,8-26,8)$ em 2002 para $26 \%(22,4-28,8)$ e $18 \%(16,4-20,6)$ em 2010, respectivamente. A taxa de redução para o sexo feminino e para o masculino foi de aproximadamente $23 \%$. Também, para ambos os sexos, verificou-se, em todos os inquéritos, que a prevalência de tabagismo diminuiu à medida que aumentou o quintil de renda e que as maiores prevalências de tabagismo ocorreram entre indivíduos com idade de 40 a 49 anos, como apresentado na análise geral.

A Tabela 3 mostra que, no sexo masculino, para cada um dos quintis ao longo dos nove anos em estudo, a redução entre aqueles pertencentes ao menor quintil de renda foi de $26 \%$, enquanto entre os do maior quintil foi de $41 \%$ (p < 0,001 ). Em relação à idade, observou-se entre os indivíduos mais jovens (20-29 anos) redução de $20 \%$, enquanto, entre os mais velhos (60 anos ou mais), de apenas $12 \%$. Estas diferenças não apresentaram $\mathrm{p}$ de tendência significativo.

Por fim, na Tabela 4, observa-se que, para o sexo feminino, a redução conforme o quintil, entre 2002 e 2010 , foi de $26 \%$ para o menor quintil de renda e de $37 \%$ no maior quintil de renda ( $\mathrm{p}<$ 0,001). Em relação à idade, houve tendência de diminuição da prevalência de tabagismo semelhante à observada na análise geral, ou seja, entre os indivíduos de 20-49 anos de idade. O teste de tendência linear de proporções apresentou valor p estatisticamente significativo para todas as reduções apresentadas.

\section{Discussão}

A comparação aqui apresentada mostrou que, entre 2002 e 2010, houve redução importante na prevalência de tabagismo em ambos os sexos, na cidade de Pelotas. Verificou-se, ainda, que, em todos os inquéritos, o tabagismo aumentou à 
Comparação de alguns indicadores entre inquéritos, 2001-2010. Pelotas, Rio Grande do Sul, Brasil.

\begin{tabular}{|c|c|c|c|c|c|}
\hline Indicador & $2001 / 2002$ & $2003 / 2004$ & $2005 / 2006$ & $2007 / 2008$ & $2009 / 2010$ \\
\hline Número de setores & 80 & 144 & 119 & 126 & 130 \\
\hline Total de domicílios & 1.600 & 1.530 & 1.597 & 1.534 & 1.512 \\
\hline Domicílios por setor & 20,0 & 10,6 & 13,4 & 12,2 & 11,6 \\
\hline Indivíduos elegíveis & 3.372 & 3.214 & 3.353 & 3.180 & 3.059 \\
\hline Período de coleta de dados & Fevereiro- & Outubro- & Outubro- & Outubro/2007- & Janeiro- \\
\hline (meses/ano) & Maio/2002 & Dezembro/2003 & Dezembro/2005 & Janeiro/2008 & Junho/2010 \\
\hline Taxa de não respondentes (\%) & 5,6 & 3,5 & 6,5 & 6,1 & 10,7 \\
\hline Amostra final & 3.182 & 3.100 & 3.136 & 2.986 & 2.732 \\
\hline Efeito de delineamento & 2,1 & 1,5 & 2,3 & 1,8 & 1,7 \\
\hline Homens na amostra (\%) & 43,2 & 43,3 & 43,9 & 43,1 & 42,1 \\
\hline Escolaridade em anos [média (DP)] & $7,4(4,3)$ & $7,7(4,4)$ & $7,9(4,6)$ & $8,2(4,7)$ & $8,2(4,6)$ \\
\hline Renda familiar em $\mathrm{R} \$$ (mediana) & 630 & 840 & 1.000 & 1.235 & 1.500 \\
\hline Idade em anos [média (DP)] & $44,0(16,3)$ & $43,2(16,1)$ & $44,1(16,4)$ & $44,8(17,0)$ & $46,1(17,0)$ \\
\hline Cor da pele branca (\%) & 84,7 & 81,0 & 84,0 & 80,1 & 81,2 \\
\hline Prevalência de tabagismo (IC95\%) & $27,9(25,6-30,2)$ & $26,6(24,7-28,6)$ & $26,7(24,3-29,1)$ & $25,7(23,6-27,8)$ & $21,3(19,3-23,3)$ \\
\hline
\end{tabular}

DP: desvio-padrão.

Associação entre renda familiar, idade e tabagismo relativamente a indivíduos com 20 anos ou mais de idade. Pelotas, Rio Grande do Sul, Brasil, 2001-2010 ( $N=15.136)$.

\begin{tabular}{|c|c|c|c|c|c|c|c|c|c|c|c|}
\hline & \multicolumn{2}{|c|}{$2001 / 2002$} & \multicolumn{2}{|c|}{$2003 / 2004$} & \multicolumn{2}{|c|}{$2005 / 2006$} & \multicolumn{2}{|c|}{$2007 / 2008$} & \multicolumn{2}{|c|}{$2009 / 2010$} & \multirow{2}{*}{$\begin{array}{c}\text { Valor de p para } \\
\text { tendência no } \\
\text { período }\end{array}$} \\
\hline & $\mathbf{n}$ & $\begin{array}{c}\text { Fumantes } \\
(\%)\end{array}$ & $\mathbf{n}$ & $\begin{array}{c}\text { Fumantes } \\
\text { (\%) }\end{array}$ & $\mathbf{n}$ & $\begin{array}{c}\text { Fumantes } \\
\text { (\%) }\end{array}$ & $\mathbf{n}$ & $\begin{array}{c}\text { Fumantes } \\
\text { (\%) }\end{array}$ & $\mathbf{n}$ & $\begin{array}{c}\text { Fumantes } \\
\text { (\%) }\end{array}$ & \\
\hline \multicolumn{12}{|l|}{ Renda familiar em quintis * } \\
\hline 1 (menor) & 615 & 38,4 & 612 & 36,4 & 637 & 36,6 & 578 & 36,5 & 541 & 28,3 & $<0,001$ \\
\hline 2 & 630 & 30,0 & 620 & 31,6 & 657 & 30,6 & 603 & 26,4 & 606 & 22,4 & $<0,001$ \\
\hline 3 & 607 & 28,2 & 604 & 25,7 & 552 & 25,2 & 577 & 26,2 & 476 & 24,4 & 0,267 \\
\hline 4 & 610 & 22,5 & 615 & 22,8 & 616 & 23,5 & 557 & 22,3 & 589 & 19,0 & 0,113 \\
\hline 5 (maior) & 612 & 21,4 & 607 & 17,3 & 612 & 17,7 & 572 & 17,8 & 491 & 13,0 & 0,002 \\
\hline $\begin{array}{l}\text { Valor de p para } \\
\text { heterogeneidade }\end{array}$ & \multicolumn{2}{|c|}{$<0,001$} & \multicolumn{2}{|c|}{$<0,001$} & \multicolumn{2}{|c|}{$<0,001$} & \multicolumn{2}{|c|}{$<0,001$} & \multicolumn{2}{|c|}{$<0,001$} & \\
\hline Valor de p para tendência & \multicolumn{2}{|c|}{$<0,001$} & \multicolumn{2}{|c|}{$<0,001$} & \multicolumn{2}{|c|}{$<0,001$} & \multicolumn{2}{|c|}{$<0,001$} & \multicolumn{2}{|c|}{$<0,001$} & \\
\hline \multicolumn{12}{|l|}{ Idade (em anos) } \\
\hline $20-29$ & 719 & 23,8 & 759 & 22,5 & 747 & 22,5 & 716 & 23,9 & 595 & 17,5 & 0,023 \\
\hline $30-39$ & 680 & 34,1 & 645 & 29,8 & 611 & 31,7 & 556 & 29,3 & 462 & 24,2 & 0,001 \\
\hline $40-49$ & 667 & 38,2 & 680 & 35,6 & 661 & 36,2 & 597 & 34,3 & 545 & 29,0 & 0,001 \\
\hline $50-59$ & 533 & 27,6 & 493 & 30,4 & 520 & 27,3 & 519 & 28,7 & 495 & 24,4 & 0,142 \\
\hline 60 ou mais & 583 & 14,1 & 523 & 13,6 & 597 & 15,9 & 598 & 13,4 & 635 & 13,9 & 0,837 \\
\hline $\begin{array}{l}\text { Valor de p para } \\
\text { heterogeneidade }\end{array}$ & \multicolumn{2}{|c|}{$<0,001$} & \multicolumn{2}{|c|}{$<0,001$} & \multicolumn{2}{|c|}{$<0,001$} & \multicolumn{2}{|c|}{$<0,001$} & \multicolumn{2}{|c|}{$<0,001$} & \\
\hline Total & 3.182 & 27,9 & 3.100 & 26,6 & 3.136 & 26,7 & 2.986 & 25,7 & 2.732 & 21,3 & $<0,001$ \\
\hline
\end{tabular}

* Sem informação para 340 (2,2\%) participantes. 
Associação entre renda familiar, idade e tabagismo relativamente a homens com 20 anos ou mais de idade. Pelotas, Rio Grande do Sul, Brasil, $2001-2010(N=6.533)$.

\begin{tabular}{|c|c|c|c|c|c|c|c|c|c|c|c|}
\hline & \multicolumn{2}{|c|}{$2001 / 2002$} & \multicolumn{2}{|c|}{$2003 / 2004$} & \multicolumn{2}{|c|}{$2005 / 2006$} & \multicolumn{2}{|c|}{$2007 / 2008$} & \multicolumn{2}{|c|}{$2009 / 2010$} & \multirow{2}{*}{$\begin{array}{c}\text { Valor de p para } \\
\text { tendência no } \\
\text { período }\end{array}$} \\
\hline & $\mathbf{n}$ & $\begin{array}{c}\text { Fumantes } \\
\text { (\%) }\end{array}$ & $\mathbf{n}$ & $\begin{array}{c}\text { Fumantes } \\
\text { (\%) }\end{array}$ & $\mathbf{n}$ & $\begin{array}{c}\text { Fumantes } \\
\text { (\%) }\end{array}$ & $\mathbf{n}$ & $\begin{array}{c}\text { Fumantes } \\
\text { (\%) }\end{array}$ & $\mathbf{n}$ & $\begin{array}{c}\text { Fumantes } \\
\text { (\%) }\end{array}$ & \\
\hline \multicolumn{12}{|l|}{ Renda familiar em quintis * } \\
\hline 1 (menor) & 249 & 45,8 & 251 & 45,0 & 260 & 41,5 & 220 & 42,7 & 213 & 33,8 & 0,008 \\
\hline 2 & 263 & 36,9 & 271 & 38,4 & 280 & 36,4 & 258 & 29,1 & 254 & 27,6 & 0,002 \\
\hline 3 & 261 & 34,1 & 267 & 30,0 & 251 & 28,3 & 256 & 28,1 & 200 & 27,0 & 0,110 \\
\hline 4 & 276 & 26,1 & 262 & 29,0 & 289 & 25,6 & 243 & 26,3 & 261 & 24,9 & 0,503 \\
\hline 5 (maior) & 272 & 24,3 & 268 & 16,4 & 270 & 20,4 & 262 & 19,8 & 209 & 14,4 & 0,058 \\
\hline $\begin{array}{l}\text { Valor de p para } \\
\text { heterogeneidade }\end{array}$ & \multicolumn{2}{|c|}{$<0,001$} & \multicolumn{2}{|c|}{$<0,001$} & \multicolumn{2}{|c|}{$<0,001$} & \multicolumn{2}{|c|}{$<0,001$} & \multicolumn{2}{|c|}{$<0,001$} & \\
\hline Valor de p para tendência & \multicolumn{2}{|c|}{$<0,001$} & \multicolumn{2}{|c|}{$<0,001$} & \multicolumn{2}{|c|}{$<0,001$} & \multicolumn{2}{|c|}{$<0,001$} & \multicolumn{2}{|c|}{$<0,001$} & \\
\hline \multicolumn{12}{|l|}{ Idade (em anos) } \\
\hline $20-29$ & 334 & 25,4 & 342 & 23,4 & 355 & 25,1 & 328 & 24,7 & 252 & 20,2 & 0,255 \\
\hline $30-39$ & 313 & 37,1 & 279 & 35,1 & 285 & 37,2 & 247 & 31,6 & 199 & 27,6 & 0,020 \\
\hline $40-49$ & 291 & 43,0 & 303 & 40,6 & 282 & 36,5 & 265 & 35,1 & 246 & 34,5 & 0,021 \\
\hline $50-59$ & 213 & 37,6 & 213 & 36,1 & 221 & 30,3 & 224 & 34,4 & 212 & 27,8 & 0,037 \\
\hline 60 ou mais & 223 & 19,7 & 207 & 20,3 & 235 & 21,7 & 222 & 18,5 & 242 & 17,4 & 0,361 \\
\hline $\begin{array}{l}\text { Valor de p para } \\
\text { heterogeneidade }\end{array}$ & \multicolumn{2}{|c|}{$<0,001$} & \multicolumn{2}{|c|}{$<0,001$} & \multicolumn{2}{|c|}{$<0,001$} & \multicolumn{2}{|c|}{$<0,001$} & \multicolumn{2}{|c|}{$<0,001$} & \\
\hline Total & 1.374 & 32,7 & 1.344 & 31,2 & 1378 & 30,2 & 1.286 & 28,8 & 1151 & 25,4 & $<0,001$ \\
\hline
\end{tabular}

* Sem informação para 167 (2,6\%) participantes.

medida que diminuiu o quintil de renda familiar e que a redução na prevalência de tabagismo ao longo deste período foi muito mais acentuada no quintil de maior renda. Por fim, as maiores prevalências concentraram-se entre indivíduos com idade de 40-49 anos.

Ao interpretar estes dados, é preciso considerar que a taxa de não respondentes praticamente duplicou ao longo do período estudado, passando de 5,6\% em 2002 para 10,7\% em 2010. Também há que se destacar que essa taxa foi ligeiramente maior entre os homens, grupo no qual a prevalência de tabagismo é maior. Isso pode ter levado a sub-registro na prevalência global de tabagismo, sobretudo no último inquérito, quando a taxa de perdas e recusas foi maior. Além disso, o uso de tabaco, tido como uma prática socialmente imprópria ou politicamente incorreta, pode ter levado alguns dos entrevistados a não informá-lo, temendo algum tipo de desaprovação. No entanto, não parece haver razão para que esta limitação tenha acometido os vários inquéritos de forma diferente.

Em um período de nove anos, a redução na prevalência de tabagismo em Pelotas foi de $23 \%$, o que implica redução anual média de 2,6\%. En- tre 1989 e 2003, dois estudos conduzidos no Brasil como um todo encontraram redução de aproximadamente $35 \%$ 6,10, enquanto na cidade de São Paulo, entre 1987 e 2002, a redução foi de $32 \% 11$. Esses percentuais representam taxa anual média de 2,5\% para o Brasil e de 2,1\% para São Paulo. Portanto, tais estudos sugerem que a prevalência de tabagismo vem caindo no país em diferentes localidades a uma taxa muito semelhante.

Apesar da redução observada, a prevalência atual de $21 \%$ para Pelotas é superior àquela encontrada pela Pesquisa Nacional por Amostras de Domicílios (PNAD) em 2008 para o Brasil $(14,5 \%)$, para a Região Sul $(16,9 \%)$ e para o Rio Grande do Sul $(18,4)$ 12. A diferença entre estes estudos diz respeito à faixa etária e à definição do desfecho. A PNAD incluiu pessoas com 15 anos ou mais, que fumavam pelo menos um cigarro por dia independentemente do tempo.

Em 2010, a prevalência de tabagismo em Pelotas foi $38 \%$ maior nos homens $(25,6 \%)$ em relação às mulheres (18,5\%). Um dos maiores e mais recentes estudos realizados no Brasil sobre tabagismo mostrou resultados semelhantes: a PNAD indicou prevalência de tabagismo de $18 \%$ entre homens contra $11 \%$ entre as mulheres para 
Associação entre renda familiar, idade e tabagismo relativamente a mulheres com 20 anos ou mais de idade. Pelotas, Rio Grande do Sul, Brasil, 2001-2010 ( $\mathrm{N}=8.603)$.

\begin{tabular}{|c|c|c|c|c|c|c|c|c|c|c|c|}
\hline & \multicolumn{2}{|c|}{$2001 / 2002$} & \multicolumn{2}{|c|}{$2003 / 2004$} & \multicolumn{2}{|c|}{$2005 / 2006$} & \multicolumn{2}{|c|}{$2007 / 2008$} & \multicolumn{2}{|c|}{$2009 / 2010$} & \multirow{2}{*}{$\begin{array}{c}\text { Valor de p para } \\
\text { tendência no } \\
\text { período }\end{array}$} \\
\hline & $\mathbf{n}$ & $\begin{array}{c}\text { Fumantes } \\
\text { (\%) }\end{array}$ & $\mathbf{n}$ & $\begin{array}{c}\text { Fumantes } \\
\text { (\%) }\end{array}$ & $\mathbf{n}$ & $\begin{array}{c}\text { Fumantes } \\
\text { (\%) }\end{array}$ & $\mathbf{n}$ & $\begin{array}{c}\text { Fumantes } \\
\text { (\%) }\end{array}$ & $\mathbf{n}$ & $\begin{array}{c}\text { Fumantes } \\
(\%)\end{array}$ & \\
\hline \multicolumn{12}{|l|}{ Renda familiar em quintis * } \\
\hline 1 (menor) & 366 & 33,3 & 361 & 30,5 & 377 & 33,2 & 358 & 32,7 & 328 & 24,7 & 0,040 \\
\hline 2 & 367 & 25,1 & 349 & 26,4 & 377 & 26,3 & 345 & 24,3 & 352 & 18,8 & 0,021 \\
\hline 3 & 346 & 23,7 & 337 & 22,3 & 301 & 22,6 & 321 & 24,6 & 276 & 22,5 & 0,996 \\
\hline 4 & 334 & 19,5 & 353 & 18,1 & 327 & 21,7 & 314 & 19,1 & 328 & 14,3 & 0,114 \\
\hline 5 (maior) & 340 & 19,1 & 339 & 18,0 & 342 & 15,5 & 310 & 16,1 & 282 & 12,1 & 0,015 \\
\hline $\begin{array}{l}\text { Valor de p para } \\
\text { heterogeneidade }\end{array}$ & \multicolumn{2}{|c|}{$<0,001$} & \multicolumn{2}{|c|}{$<0,001$} & \multicolumn{2}{|c|}{$<0,001$} & \multicolumn{2}{|c|}{$<0,001$} & \multicolumn{2}{|c|}{$<0,001$} & \\
\hline Valor de p para tendência & \multicolumn{2}{|c|}{$<0,001$} & \multicolumn{2}{|c|}{$<0,001$} & \multicolumn{2}{|c|}{$<0,001$} & \multicolumn{2}{|c|}{$<0,001$} & \multicolumn{2}{|c|}{$<0,001$} & \\
\hline \multicolumn{12}{|l|}{ Idade (em anos) } \\
\hline $20-29$ & 385 & 22,3 & 417 & 21,8 & 392 & 20,1 & 388 & 23,2 & 343 & 15,4 & 0,047 \\
\hline $30-39$ & 367 & 31,6 & 366 & 25,7 & 326 & 27,0 & 309 & 27,5 & 263 & 21,7 & 0,030 \\
\hline $40-49$ & 376 & 34,6 & 377 & 31,6 & 379 & 35,9 & 332 & 33,7 & 299 & 24,4 & 0,019 \\
\hline $50-59$ & 320 & 21,0 & 280 & 26,1 & 299 & 25,1 & 295 & 24,4 & 283 & 21,9 & 0,871 \\
\hline 60 ou mais & 360 & 10,6 & 316 & 9,2 & 362 & 12,1 & 376 & 10,4 & 393 & 11,7 & 0,483 \\
\hline $\begin{array}{l}\text { Valor de p para } \\
\text { heterogeneidade }\end{array}$ & \multicolumn{2}{|c|}{$<0,001$} & \multicolumn{2}{|c|}{$<0,001$} & \multicolumn{2}{|c|}{$<0,001$} & \multicolumn{2}{|c|}{$<0,001$} & \multicolumn{2}{|c|}{$<0,001$} & \\
\hline Total & 1.808 & 24,2 & 1.756 & 23,1 & 1.758 & 24,0 & 1.700 & 23,4 & 1.581 & 18,4 & $<0,001$ \\
\hline
\end{tabular}

* Sem informação para 173 (2,0\%) participantes.

o país. Para o Estado do Rio Grande do Sul, este mesmo estudo mostrou prevalência de $20 \%$ entre os homens e de $14 \%$ entre as mulheres 12 .

O percentual de redução do tabagismo, no presente estudo, foi de $23 \%$ entre os homens e $24 \%$ entre as mulheres, resultado ligeiramente diferente daquele encontrado por Monteiro et al. 6 para o país como um todo. Na pesquisa destes autores, que se referiu a um período de 15 anos, de 1989 a 2003, a redução foi de $37 \%$ entre os homens e de $32 \%$ entre as mulheres. Em um período semelhante (1987-2002), Marcopito et al. 11 identificaram, na cidade de São Paulo, redução de $39 \%$ no tabagismo para o sexo masculino e de $35 \%$ para o feminino. Essa diferença quanto à redução conforme o sexo em relação aos dois outros estudos brasileiros pode ser decorrente do periodo de coleta de dados (defasagem de oito anos), da faixa etária estudada (um pouco mais ampla) e da definição de desfecho (não consideraram tempo mínimo para determiná-lo).

Trabalhos recentes conduzidos em outros países mostraram reduções maiores na prevalência de tabagismo entre as mulheres, quando comparadas às observadas no presente estudo. $\mathrm{Na}$ Coréia do Sul, investigação realizada entre 1995 e
2006 mostrou redução de $24 \%$ entre os homens e de $22 \%$ entre as mulheres. A faixa etária incluída no estudo sul-coreano era de 25-64 anos 13 . Similarmente, em Minnesota, Estados Unidos, entre 1980 e 2000, verificou-se redução de $39 \%$ entre os homens e $37 \%$ entre as mulheres, o que representa redução anual média de cerca de $2 \% 14$.

O presente estudo mostrou clara tendência de aumento da prevalência de tabagismo à medida que diminuía a renda em todos os inquéritos e, entre 2002 e 2010, maior redução entre aqueles pertencentes ao maior quintil de renda. Nos estágios iniciais da epidemia de tabagismo, este esteve mais presente entre os indivíduos de melhor nível socioeconômico. Era um indicador de posição social, de status quo. À medida que a epidemia avançou, houve uma inversão, e o tabagismo passou a ser mais comum entre os mais pobres 15,16. A maior redução observada neste estudo entre os indivíduos de maior renda coincide com os padrões da epidemia propostos por Lopez et al. 16 em 1994 para países desenvolvidos. Segundo os autores 16, indivíduos com nível socioeconômico mais alto seriam mais susceptíveis a campanhas sobre os riscos do tabagismo. Tais achados são reforçados por Monteiro et al. ${ }^{6}$, que 
observaram associação direta entre poder aquisitivo e intensidade de redução na prevalência de fumantes para ambos os sexos. Em um estudo realizado nos Estados Unidos entre 1990 e 2004, verificou-se declínio nas prevalências médias de tabagismo, sendo este maior entre aqueles de melhor nível socioeconômico 17.

Foi observado, no presente estudo, para os sexos masculino e feminino, aumento das prevalências de tabagismo até a faixa etária de 40-49 anos, e posterior declínio. Não foram encontrados trabalhos que explicassem esse comportamento em todos os inquéritos. Seria esperado que as maiores prevalências tendessem a acometer as faixas etárias mais velhas com o passar dos anos, o que não foi observado. Dessa maneira, enfatizamos a necessidade de estudos que abordem estes achados com maior detalhamento.

Esta pesquisa mostrou diminuição significativa na prevalência do tabagismo entre indivíduos do sexo masculino com idade entre $30 \mathrm{e}$ 60 anos, mas não nas faixas etárias extremas. Já no sexo feminino, a tendência de diminuição foi significativa dos 20 aos 49 anos de idade. Estudo realizado na Coréia do Sul demonstrou diminuição em todas as faixas etárias para o sexo masculino, enquanto, no sexo feminino, observou-se diminuição apenas entre aquelas com 45 anos ou mais de idade e aumento na prevalência de tabagismo entre as mais jovens (20-24 e 25-44 anos) 18 .

A redução encontrada neste estudo pode ser resultado de um conjunto de ações e medidas instituídas no Brasil e, particularmente, no Município de Pelotas ao longo do período estudado. Em nível nacional, os maços de cigarros passaram a conter imagens que mostram o efeito devastador do tabagismo sobre o organismo; foi instituído o serviço Disque Pare de Fumar; a rede pública passou a oferecer tratamento para os fumantes; o preço real do cigarro aumentou e as propagandas nos meios de comunicação passaram a sofrer restrições, além da completa proibição em eventos culturais e esportivos. $\mathrm{O}$ impacto dessas iniciativas sobre a prevalência de tabagismo não foi ainda adequadamente avaliado no Brasil, no entanto estudo conduzido no Canadá mostrou que as imagens são efetivas na cessação do tabagismo 19. A busca por auxilio para deixar de fumar através do Disque Pare de Fumar aumentou em cerca de quatro vezes desde sua divulgação nos maços de cigarros a partir de 2002 20. Em Pelotas, em 2009, foi aprovada lei municipal proibindo totalmente o fumo em ambientes coletivos. Embora o impacto desta medida sobre o tabagismo não esteja ainda bem estabelecido, é possível assegurar que ela reduz a exposição ao fumo passivo e inibe o ato de fu$\operatorname{mar} 21,22$.

A diminuição na prevalência de tabagismo é evidente em ambos os sexos. Os de menor renda familiar foram os que menos se beneficiaram desta redução, a qual pode ser decorrente de medidas de apoio aos que desejam deixar de fumar, aumento do preço dos cigarros, regulação e proibição da propaganda de cigarro e a exclusão do fumo de ambientes coletivos. No entanto, esses fatores precisam ser detalhadamente investigados.

Por fim, não obstante o declínio observado, a prevalência de tabagismo na cidade de Pelotas continua elevada. Esse fato mostra a necessidade de fortalecer as ações em curso e de direcioná-las àqueles de menor renda familiar, de ambos os sexos, a fim de alcançar um maior impacto em um menor período de tempo. 


\section{Resumo}

Avaliou-se a tendência temporal da prevalência de tabagismo conforme renda familiar, idade e sexo entre indivíduos com 20 anos ou mais residentes em Pelotas, Rio Grande do Sul, Brasil. Cinco inquéritos de base populacional utilizando-se de setores censitários foram realizados na cidade entre 2002-2010. Considerouse tabagismo o consumo de um ou mais cigarros por dia há pelo menos um mês. A tendência temporal foi avaliada por meio do teste de qui-quadrado para tendência linear. Os cinco inquéritos realizados incluíram 15.136 indivíduos. Neste período, a prevalência total de tabagismo caiu de 28\% (25,8-30,4) em 2002 para $21 \%(19,5-23,5)$ em 2010. Esta queda de 23\% foi semelhante entre os sexos, mas muito diferente em relação à renda familiar. Quanto menor a renda familiar, maior a prevalência de tabagismo. Entre 2002-2010, a taxa de redução do tabagismo foi de $26 \%$ no menor quintil de renda e de 39\% no maior. Apesar das reduções observadas, a prevalência de tabagismo ainda é elevada, indicando a necessidade de fortalecer medidas de controle voltadas principalmente aos indivíduos com menor renda.

Tabagismo; Inquéritos Epidemiológicos; População Urbana; Prevalência

\section{Referências}

1. World Health Organization. Building blocks for tobacco control: a handbook. Geneva: World Health Organization; 2004.

2. World Health Organization. Report on the Global Tobacco Epidemic, 2011: warning about the dangers of tobacco. Geneva: World Health Organization; 2011.

3. Pinto M, Ugá M. Os custos de doenças tabacorelacionadas para o Sistema Único de Saúde. Cad Saúde Pública 2010; 26:1234-45.

4. Corrêa P, Barreto S, Passos V. Smoking-attributable mortality and years of potential life lost in 16 Brazilian capitals, 2003: a prevalence-based study. BMC Public Health 2009; 9:206.

5. Mackay J, Eriksen M. The tobacco atlas. Geneva: World Health Organization; 2002.

6. Monteiro C, Cavalcante T, Moura E, Claro R, Szwarcwald C. Population-based evidence of a strong decline in the prevalence of smokers in Brazil (1989-2003). Bull World Health Organ 2007; 85:527-34

\section{Colaboradores}

J. L. Dias-Damé elaborou o projeto, coordenou a coleta de dados, realizou análise preliminar e final dos dados e redigiu o artigo. J. A. Cesar auxiliou na elaboração do projeto, na análise de dados e na revisão final do artigo. S. M. Silva auxiliou na elaboração do instrumento de coleta, na preparação do banco de dados e na redação final do artigo.
7. Berg CM, Lissner L, Aires N, Lappas G, Toren K, Wilhelmsen L, et al. Trends in blood lipid levels, blood pressure, alcohol and smoking habits from 1985 to 2002: results from INTERGENE and GOT-MONICA. Eur J Cardiovasc Prev Rehabil 2005; 12:115-25.

8. Barros A, Menezes A, Santos I, Assunção M, Gigante D, Fassa A, et al. O Mestrado do Programa de Pós-graduação em Epidemiologia da UFPel baseado em consórcio de pesquisa: uma experiência inovadora. Rev Bras Epidemiol 2008; 11:133-44.

9. World Health Organization. Definitions of smoking. Global link. Geneva: World Health Organization; 2003.

10. Figueiredo VC. Um panorama do tabagismo em 16 capitais brasileiras e Distrito Federal: tendências e heterogeneidades [Tese de Doutorado]. Rio de Janeiro: Universidade do Estado do Rio de Janeiro; 2007. 
11. Marcopito L, Coutinho A, Valencich D, Moraes M, Brumini R, Ribeiro S. Exposição ao tabagismo e atitudes: comparação entre inquéritos realizados na população adulta do município de São Paulo em 1987 e 2002. Arq Bras Cardiol 2007; 89:333-40.

12. Instituto Brasileiro de Geografia e Estatística. Pesquisa Nacional por Amostra de Domicílios (PNAD). Tabagismo, 2008. http://www.ibge.gov. $\mathrm{br} /$ home/estatistica/populacao/trabalhoerendi mento/pnad2008/suplementos/tabagismo/pnad tabagismo.pdf (acessado em 13/Nov/2010).

13. Khang Y, Yun S, Cho H, Jung-Choi K. The impact of governmental antismoking policy on socioeconomic disparities in cigarette smoking in South Korea. Nicotine Tob Res 2009; 11:262.

14. Duval S, Jacobs Jr. DR, Barber C, Lando HA, Steffen LM, Arnett DK, et al. Trends in cigarette smoking: the Minnesota Heart Survey, 1980-1982 through 2000-2002. Nicotine Tob Res 2008; 10:827-32.

15. Cavelaars A, Kunst A, Geurts J, Crialesi R, Grotvedt L, Helmert U, et al. Educational differences in smoking: international comparison. BMJ 2000; 320:1102.

16. Lopez A, Collishaw N, Piha T. A descriptive model of the cigarette epidemic in developed countries. Tob Control 1994; 3:242-7.

17. Harper S, Lynch J. Trends in socioeconomic inequalities in adult health behaviors among US states, 1990-2004. Public Health Rep 2007; 122:177.
18. Khang Y, Cho H. Socioeconomic inequality in cigarette smoking: trends by gender, age, and socioeconomic position in South Korea, 1989-2003. Prev Med 2006; 42:415-22.

19. Hammond D, Fong GT, McDonald PW, Cameron R, Brown KS. Impact of the graphic Canadian warning labels on adult smoking behaviour. Tob Control 2003; 12:391-5.

20. Departamento de Ouvidoria Geral do SUS, Secretaria de Gestão Estratégica e Participativa, Ministério da Saúde/Instituto Nacional de Câncer. Apresentação de dados do serviço Disque Saúde. Opção: como parar de fumar. http://portal.saude.gov. $\mathrm{br} /$ portal/arquivos/pdf/relatorio_tematico_\%20 inca.pdf (acessado em 14/Nov/2010).

21. Edwards R, Thomson G, Wilson N, Waa A, Bullen C, O'Dea D, et al. After the smoke has cleared: evaluation of the impact of a new national smoke-free law in New Zealand. Tob Control 2008; 17:e2.

22. Regidor E, de Mateo S, Ronda E, Sánchez-Payá J, Gutiérrez-Fisac J, de la Fuente L, et al. Heterogeneous trend in smoking prevalence by sex and age group following the implementation of a national smoke-free law. J Epidemiol Community Health 2010; 65:702-8.

Recebido em 08/Mai/2011

Versão final reapresentada em 12/Jul/2011

Aprovado em 18/Jul/2011 